\title{
Improving teacher awareness through activity, badge and content visualizations
}

\author{
Sven Charleer, Jose Luis Santos, Joris Klerkx, and Erik Duval \\ Dept. of Computer Science, KU Leuven \\ Leuven, Belgium \\ \{Sven.Charleer, JoseLuis. Santos, Joris.Klerkx, Erik. Duval\}@cs .kuleuven . be
}

\begin{abstract}
This paper introduces LARAe (Learning Analytics Reflection \& Awareness environment), a teacher-oriented dashboard that visualizes learning traces from students, badges and course content. We also present an evaluation of the dashboard in a course on Human-Computer Interaction. The LARAe teacher dashboard provides a detailed overview of group and individual activities, achievements and course outcomes. To help visualize the abundance of traces, badges are used to abstract essential aspects of the course such as course goals and social activity. This paper reports our work on LARAe, presents the course in which we evaluated our approach with students and teachers, and analyses our first results that indicate that such an environment can help with teacher awareness.
\end{abstract}

Keywords: learning analytics, learning dashboards, collaboration, reflection, awareness, information visualization, open badges

\section{Introduction}

Feedback and collaborative discourse, between student and teacher, among students and even with external parties, leads to significant gains in learning [1]. Traditional tools for such discourse are exams, discussion fora, self-assessment and peer evaluations, but also (micro-)blogging (Twitter, Wordpress, Facebook) can help students share and reflect on their work, collaborate, discuss and learn from peers [7]. These activities leave behind a multitude of learner traces that reflect progress of students [17]. Reflecting on those traces can help learners to understand what is the optimal setting and context in which they learn best. Teachers on the other hand can, among other things, use those traces to find out which student is struggling with what content. Information visualization offers effective ways to explore this abundance of data, find new insights, and tell compelling stories.

In previous work, we have developed visualization tools such as StepUp!, SAM and TinyARM [17]. These tools used basic graphical representations such as bar charts, line charts and parallel coordinates to present all traces of all users. Evaluations have shown that they can provide a broad insight on student activities, but also that the abundance of information is overwhelming for students [13]. 
Our work presented in this paper goes one step further and provides students and teachers with Open Badges ${ }^{1}$ as abstractions of essential aspects of the course such as achieving particular course goals or social activity. This paper introduces LARAe, a large display visualization for teachers that combines these abstractions through badges with its underlying raw data (e.g. amount and content of blogposts of a student) to provide deep-level awareness of all student activities, achievements and outcomes.

The remainder of this paper is structured as follows: section 2 discusses relevant examples of learning dashboards and the use of badges in learning. The context of our evaluation, including course design and badge methodology, is presented in section 3. Section 4 then elaborates on the LARAe dashboard. Section 5 presents preliminary results of the evaluation, followed by a discussion on improvements and conclusions in section 6 .

\section{Background \& Related Work}
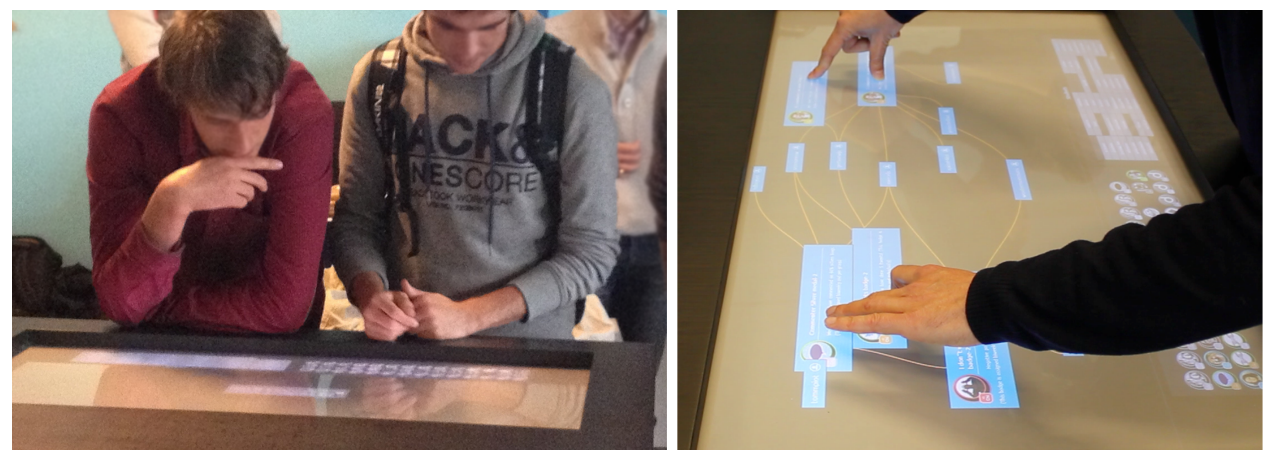

Fig. 1. An interactive tabletop application allowing collaborative reflection through badge visualizations. The lack of content and context makes it difficult to interpret the data.

Similar to the Quantified Self ${ }^{2}$ movement, which focuses on collecting user traces and using the data for self-improvement, Learning Analytics can help understand and optimize learning and the environments in which it occurs [15]. By tracking learner activities (which in our context include software development with a shared source repository, blogging, time tracking, posting on a discussion forum, etc.), these learning traces [4] can be visualized on interactive dashboards, helping students and teachers become more aware of their activities [13].

These visualizations can help teachers evaluate and improve course activities, structure and materials $[11,12]$. The data is also valuable for detecting and

\footnotetext{
${ }^{1}$ http://openbadges.org

2 http://quantifiedself.com
} 
predicting problematic students [18] or imbalanced group activity [8], allowing for quick intervention.

These data can be presented in many ways [16]. Badges, which can represent abstractions of learning traces, bring with them many benefits and uses: The creation process of the badges can influence the design of the course [6] and hence create clearer goals for both student and teacher. Badges can be used as feedback and are proven to directly impact behavior and motivate students in off- and online courses [6], [9], [13]. Skill recognition can be brought outside the classroom to support life long learning by using badges as certifications in e.g. Massive Open Online Courses [5,6].

The meaning of data might get lost through abstraction. Teachers and students can misinterpret the information when it is limited to e.g. activity count, grades and course goals. Previous work [3] has shown that there is a need for context and content to enrich the badge data (Figure 1). Following the visual information-seeking mantra of "Overview first, zoom and filter, then details-ondemand" [14], the dashboard presented in this paper uses both badges and an overview of activity to give teachers a gateway to the content, retaining a sense of context and providing access to the details.

\section{Case: Course on HCI}

We evaluated our LARAe dashboard following a user-centered rapid prototyping approach, where we first rely on paper prototypes to gather initial feedback on early ideas and then gradually develop more functional digital prototypes in rapid iteration cycles. The concrete evaluation context was a course on HumanComputer Interaction (HCI) for computer science master students, taught in 2013, with 26 students.

\subsection{Course environment}

The course focused on the design, development and evaluation of a recommendation application, i.e. a tool that enables humans to recommend resources to other humans (as opposed to recommender systems). It was taught to 26 engineering students between the ages of 20 and 25. Students worked in groups of 3 and improved their application through iterative development. The course includes face-to-face studio sessions. All presentations, course material and reports are publicly accessible online, through Slideshare, the course wiki ${ }^{3}$ and the student group blogs. Twitter with a course specific hashtag (\#chikul13) is used to share opinions, questions and comments about the course. In total, 142 blog posts, 549 blog comments and 548 tweets were generated during the course.

\subsection{Capturing and Exposing Learning Traces}

Our back-end system consists of a tracking service, Data Store and Badge Rewarding System.

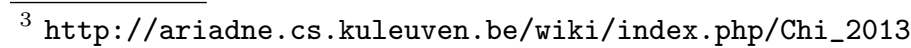


To collect Learning Analytics data, the tracking service collects blog posts and comments from the course blogs through their $\mathrm{RSS}^{4}$ feeds. It connects to the Twitter $\mathrm{API}^{5}$, to collect course related tweets which are identified by the \#chikul13 hashtag. The tracking service supports multiple sources, from RSS feeds to proprietary APIs such as the weSPOT Inquiry system ${ }^{6}$ [10].

These learning traces are stored in the Data Store which also exposes the data through a RESTful web service [13].

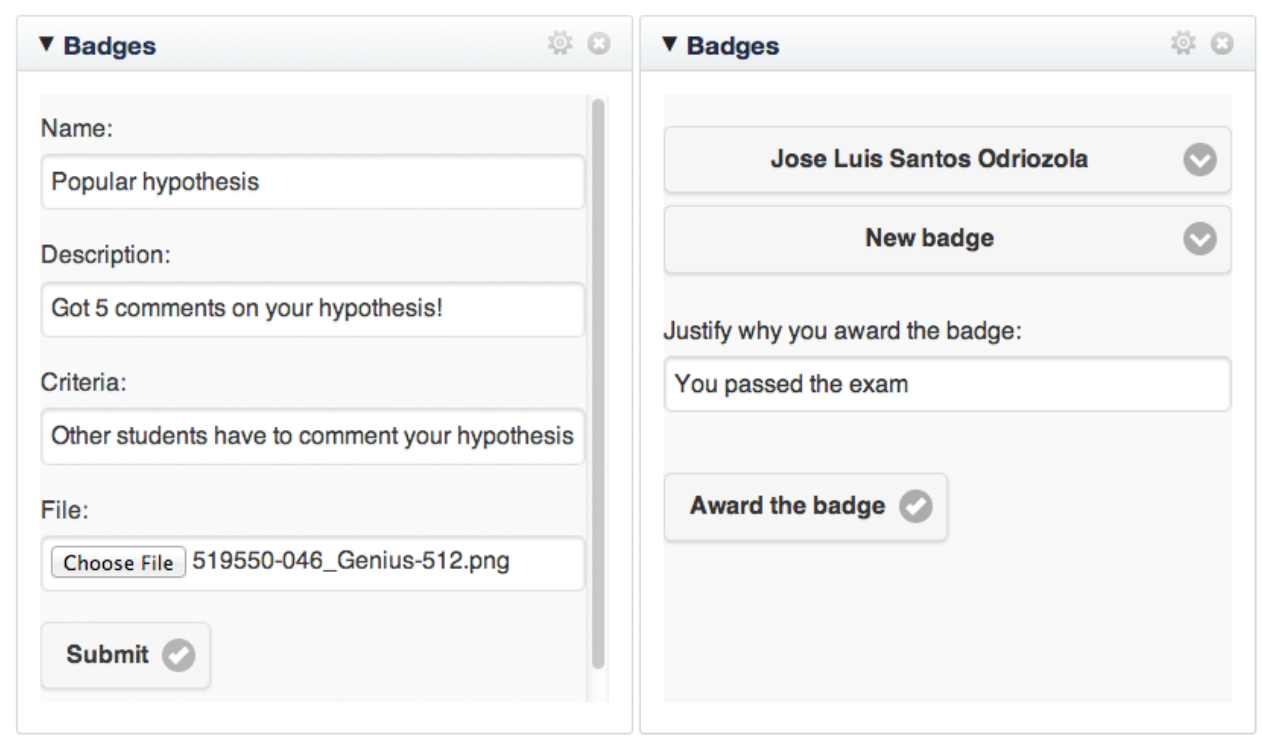

Fig. 2. Example of the Badge Award widget for weSPOT Inquiry system. Left: Creation of a badge. Right: Awarding a badge to a student.

The Badge Rewarding System allows for both automatic and manual assignment of badges to students. The Badge $\mathrm{API}^{7}$ facilitates the development of applications on top of the Badge Rewarding System, e.g. applications for teachers to define and assign badges (Figure 2). The source code is available at https:// github.com/jlsantoso/stepup/tree/chicourse/OpenBadgesAPI. Section 3.3 will further explain the badges deployed in the HCI course and the automatic awarding of badges.

\footnotetext{
${ }^{4}$ http://en.wikipedia.org/wiki/Rss

${ }^{5}$ https://dev.twitter.com/docs/api

6 http://inquiry.wespot.com

7 http://wespot.net/en/apis
} 


\subsection{Abstracting Traces through Badges}

Summarizing data and relating them to important aspects, such as intended learning outcomes, can be achieved with badges. To define what badges we use in our HCI course, we worked together with the teacher to identify the most important activities. Blogs and Twitter play a big role in the course as they are indicators of commitment and collaboration, so we want to reward posting of tweets and blog posts. For example, badges are awarded for a specific number of tweets, posts, and comments. Badges can also indicate a certain level of quality of the content of a blog post which can be derived from the number of comments it receives, by students, teachers and externals participants in the course (This particular course follows an open approach that enables anyone to comment on the content, discussions, activities and results in the course). Inactivity is a behavior that students should avoid. This is translated into a negative badge. Furthermore, some badges are allocated individually and some are assigned to teams. In total, 51 different kinds of badges are defined. The full list together with an explanation of their meaning can be found at http://navi-hci.appspot. com/badgeoverview.

Badges are automatically assigned bi-weekly. Certain activity in the tracked data will trigger the assignment of a badge when requirements are met and a mail is sent to the student with information on the awarded badge. This badge data is also stored and accessible through a REST service, creating an open data framework on which other tools can easily be developed. Furthermore, we follow the Mozilla Open Badges Standard ${ }^{8}$ for describing the badges, so that our framework is interoperable with other systems such as the Learning Management Systems Moodle ${ }^{9}$ and Blackboard ${ }^{10}$. On top of that, students can choose to publish their awards on social networks if they like to do so.

\section{The LARAe Dashboard}

On top of these services, we develop our learning dashboards to deliver visualizations that help get insights about how learners interact with content and with other learners, teams and external users.

The dashboard is presented on a large desktop, an interactive whiteboard or a touch display and consists of 6 main information areas (see Figures 3 and 4):

1. Student-Badge Matrix: With student names on the horizontal axis and badges on the vertical axis, the matrix gives an overview of how many times a specific student has been awarded a specific badge. Larger circles denote that a badge has been awarded more often to a particular student.

2. Activities/Badges Over Time: This view consists of 5 graphs. The first graph displays the total activity of all students over time by day. These

\footnotetext{
${ }^{8}$ http://openbadges.org

${ }^{9}$ http://moodle.org

10 http://blackboard.com
} 


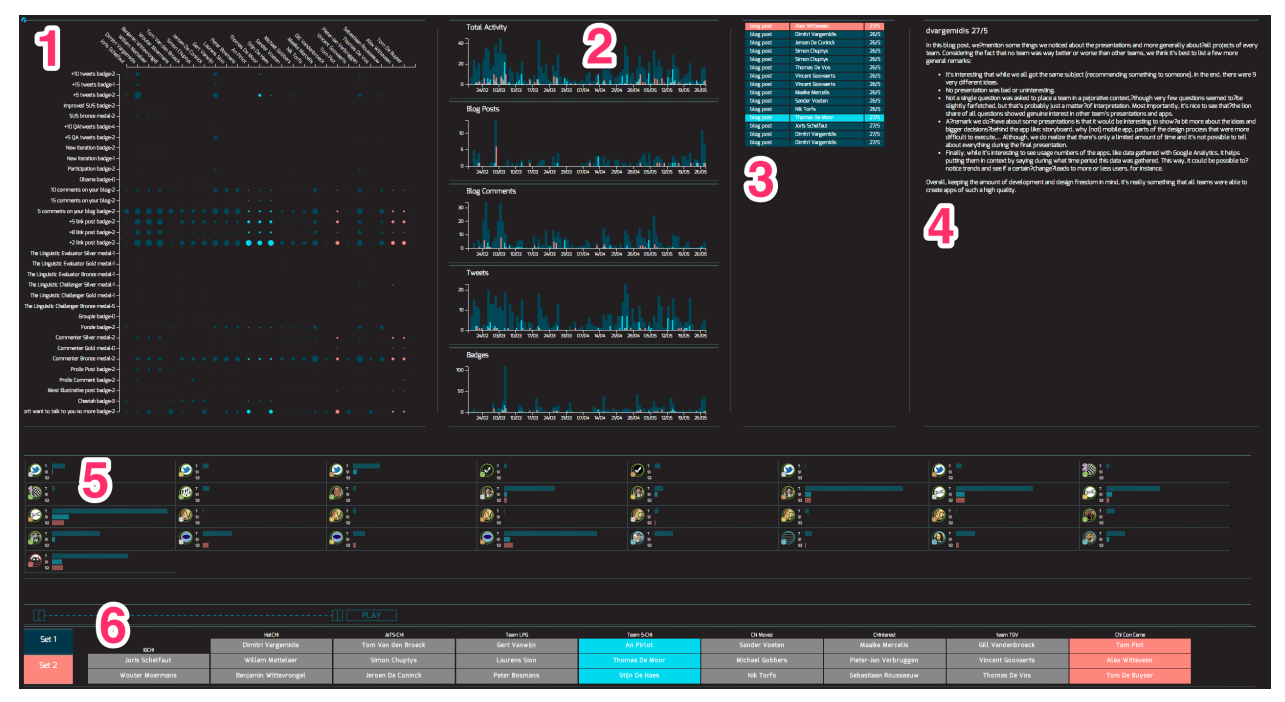

Fig. 3. Overview of information areas of the LARAe dashboard.
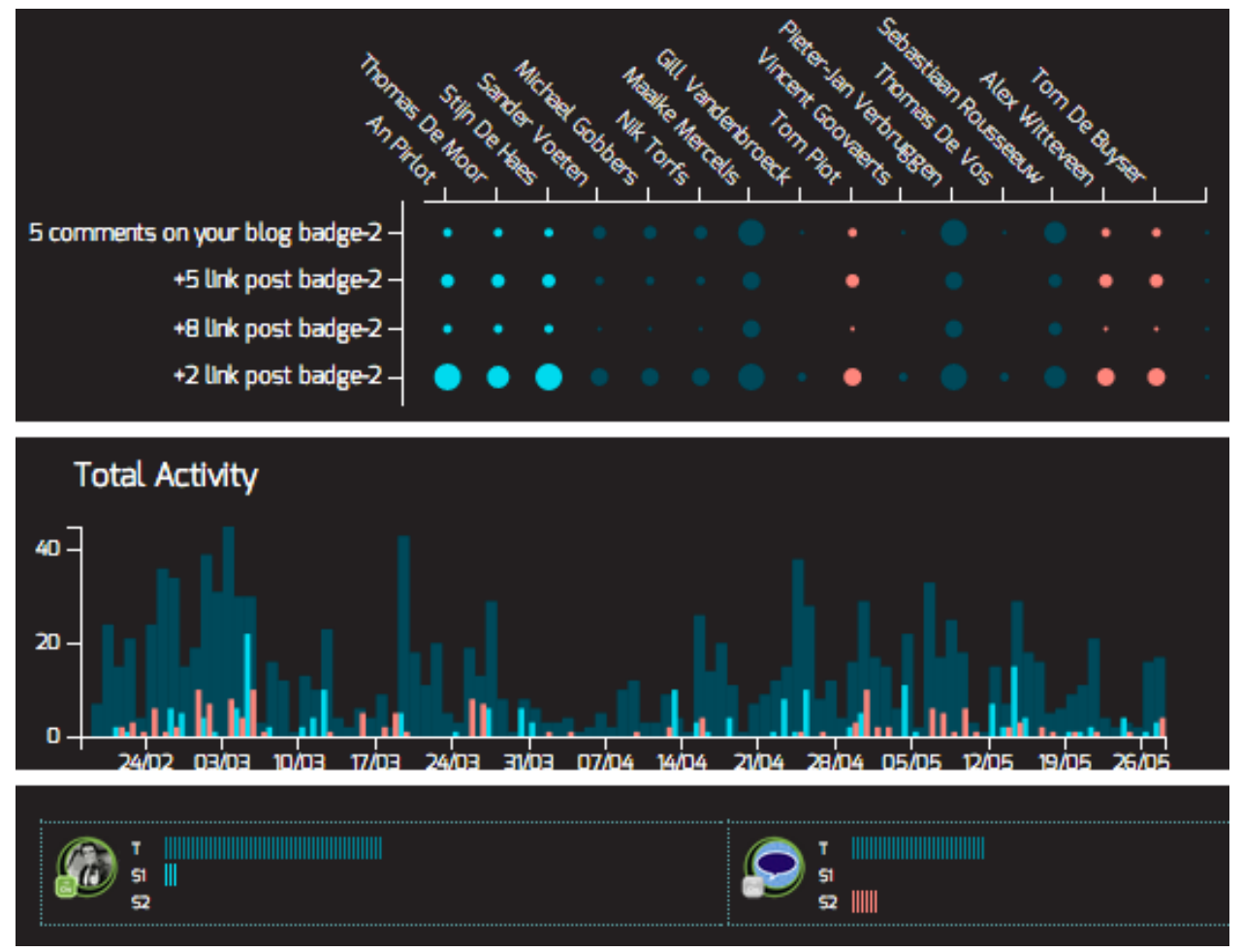

Fig. 4. Zooming in on the Student-Badge Matrix, Activities Over Time and Badge Overview graphs. 
activities are split up in the next 3 graphs: blog posts, blog comments and tweets. The last graph shows the number of badges awarded each day. The bars of these bar charts are interactive. Clicking or touching a bar will update the Activity List.

3. Activity List: This list contains the activities done or badges awarded on the selected day of the bar chart. These items are selectable and will update the Activity Details Field.

4. Activity Details Field: This fields shows the content that is linked to an activity. In the case of blog activity, it will provide the user with the content of the blog post or comment. A tweet activity will display the related tweet. The field can also present more information on badges, such as the name and description.

5. Badge Overview: The Badge Overview is another visualization of the awarded badges and facilitates student or group comparison (see Figure 4 for details). T represents the total number of badges awarded to the class. S1 and S2 stand for Set 1 and Set 2 and display the number of badges awarded to the sets of selected students (see Filter Area). Clicking on a badge provides the user with a description of the badge.

6. Filter Area: A number of filtering options are available in the Filter Area. Students can be selected from the list and can be assigned to Set 1 (blue) and Set 2 (red). All other areas will be updated with the cumulative data of the selected students in each set, in the corresponding set color (see Figure 4): the Student-Badge matrix will highlight the selected student, the Activities Over Time will show the subset of data as an overlay on top of the total data, the Badge Overview will show the total number of badges awarded in Set 1 and Set 2, and the Activity List will highlight activities done by the selected students. The time slider allows the user to modify the time range of the data displayed on the dashboard.

The dashboard is a web application developed using HTML5, Javascript, D3.js ${ }^{11}$ and crossfilter.js ${ }^{12}$. The backend is created with Node.js ${ }^{13}$ and MongoDB ${ }^{14}$.

\section{Preliminary Evaluation Results}

Our initial evaluations serve to gather feedback about perceived usefulness and effectiveness of the dashboard. We also wish to understand how users interacts with the visualization e.g. which information areas attract most attention and why, whether the visualization can provide insights and if badges enriched with context and content prove to be an added value for teachers.

We evaluated LARAe with 6 people with teaching responsibilities. 3 out of 6 participants were involved in the teaching of the HCI course. Participants used a 27" high resolution screen and a trackpad to interact with the dashboard.

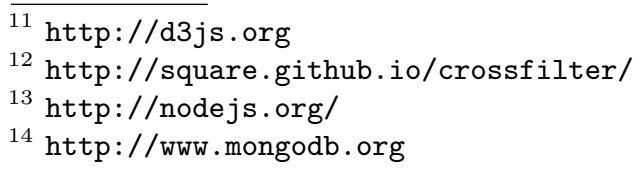


Using the think-aloud protocol, the participants were asked to explore the different information areas of the dashboard. They freely interacted with the visualizations and attempted to make sense of the data. Once a participant got stuck, a scenario was provided to explore the dashboard further. The evaluation ended with 49 5-scale Likert questions (1 - Strongly disagree, 5 - Strongly agree) covering clarity, usefulness and effectiveness of the different information areas and a SUS questionnaire.

The SUS score was $76(\mathrm{SD}=7)$ [2]. This matches the observations during the evaluations: 5 out of 6 participants understood each information area without help and covered all scenarios without external input. From the survey, questions about the general clarity of each area were all rated positively $(\mathrm{M}=4.0, \mathrm{SD}=$ $0.9)$.

Participants found the ability to compare students and groups of students very interesting. Survey results averaged $4.0(\mathrm{SD}=0.6)$ for perceived effectiveness regarding activity comparison and $3.5(\mathrm{SD}=0.8)$ regarding badge comparison. This sparked interest for a deeper analysis and led users to search for links between activities, badges and grades. The comparison was usually done between the best and lowest graded groups (scores were presented next to student names, omitted from Figure 3 for student privacy). The survey revealed that most users considered LARAe useful for understanding class achievements and activities $(\mathrm{M}=3.8, \mathrm{SD}=0.8)$; participants started their analysis by exploring the Student-Badge Matrix, typically by first looking for high and low achievers. The Activity Over Time graphs were used afterwards to analyze the activity and recognize periods of student/group inactivity .

All participants agreed that the dashboard improves their general awareness of the activities $(\mathrm{M}=4.0, \mathrm{SD}=0.6)$ and the badges awarded $(\mathrm{M}=4.1, \mathrm{SD}=$ 0.4 ). Because it provides access to activity, achievement and student-generated data (blog posts, comments, tweets), the dashboard was considered beneficial to use in discussions and evaluations of course outcomes among teaching staff (M $=4.0, \mathrm{SD}=0.9)$ and with students $(\mathrm{M}=4.0, \mathrm{SD}=0.6)$.

\section{Conclusion \& Future work}

Learning dashboards provide a means of visualizing the abundance of learner traces that are left by students and teachers during a course. We presented our experiment of simplifying the data through badges that emphasize the more important student activities and course goals. We also explained how the LARAe dashboard improves teacher awareness of activity and achievements. As the dashboard was perceived useful as an evaluation and discussion tool, we will perform further evaluations using more collaborative settings (e.g. interactive tabletops, interactive whiteboards), among teachers but also together with students.

While LARAe already facilitates access to the student-generated content (blog posts, comments and tweets), we believe visualizing the learning traces through abstractions like badges and using them as a gateway to content can help both teachers and students with the task of keeping track of and learning 
from the large amount of data they generate. We will therefore further research how we can improve teacher and student awareness regarding outcomes.

Acknowledgements. The research leading to these results has received funding from the European Community's Seventh Framework Programme (FP7/20072013) under grant agreement No 318499 - weSPOT project.

\section{References}

1. Black, P., Wiliam, D.: Assessment and classroom learning. Assessment in Education: Principles, Policy \& Practice 5(1), 7-74 (1998)

2. Brooke, J.: Sus - a quick and dirty usability scale. Usability evaluation in industry 189, 194 (1996)

3. Charleer, S., Klerkx, J., Santos, J.L., Duval, E.: Improving awareness and reflection through collaborative, interactive visualizations of badges. In: Kravcik, M., Krogstie, B.R., Moore, A., Pammer, V., Pannese, L., Prilla, M., Reinhardt, W., Ullmann, T.D. (eds.) ARTEL@EC-TEL. CEUR Workshop Proceedings, vol. 1103, pp. 69-81. CEUR-WS.org (2013)

4. Duval, E., Verbert, K.: Learning analytics. E-Learning and Education 1(8) (2012)

5. Goligoski, E.: Motivating the learner: Mozilla's open badges program. Access to Knowledge: A Course Journal 4(1) (2012)

6. Higashi, R., Abramovich, S., Shoop, R., Schunn, C.: The roles of badges in the computer science student network. In: 2012 Games+Learning+Society Conference. Citeseer (2012)

7. Lin, W.J., Liu, Y.L., Kakusho, K., Yueh, H.P., Murakami, M., Minoh, M.: Blog as a tool to develop e-learning experience in an international distance course. In: Sixth International Conference on Advanced Learning Technologies. pp. 290-292. IEEE (2006)

8. Martinez Maldonado, R., Kay, J., Yacef, K., Schwendimann, B.: An interactive teacher's dashboard for monitoring groups in a multi-tabletop learning environment. In: Cerri, S., Clancey, W., Papadourakis, G., Panourgia, K. (eds.) Intelligent Tutoring Systems, LNCS, vol. 7315, pp. 482-492. Springer Berlin Heidelberg (2012)

9. McDaniel, R., Lindgren, R., Friskics, J.: Using badges for shaping interactions in online learning environments. In: IEEE International Professional Communication Conference. pp. 1-4. IEEE (2012)

10. Mikroyannidis, A., Okada, A., Scott, P., Rusman, E., Specht, M., Stefanov, K., Boytchev, P., Protopsaltis, A., Held, P., Hetzner, S., Kikis-Papadakis, K., Chaimala, F.: wespot: A personal and social approach to inquiry-based learning. Journal of Universal Computer Science 19(14), 2093-2111 (2013)

11. Raca, M., Dillenbourg, P.: System for assessing classroom attention. In: Proceedings of the Third International Conference on Learning Analytics and Knowledge. pp. 265-269. LAK '13, ACM, New York, NY, USA (2013)

12. Rivera-Pelayo, V., Munk, J., Zacharias, V., Braun, S.: Live interest meter: Learning from quantified feedback in mass lectures. In: Proceedings of the Third International Conference on Learning Analytics and Knowledge. pp. 23-27. LAK '13, ACM, New York, NY, USA (2013) 
13. Santos, J., Charleer, S., Parra, G., Klerkx, J., Duval, E., Verbert, K.: Evaluating the use of open badges in an open learning environment. In: Hernández-Leo, D., Ley, T., Klamma, R., Harrer, A. (eds.) Scaling up Learning for Sustained Impact, LNCS, vol. 8095, pp. 314-327. Springer Berlin Heidelberg (2013)

14. Shneiderman, B.: The eyes have it: a task by data type taxonomy for information visualizations. In: IEEE Symposium on Visual Languages. pp. 336-343. IEEE (1996)

15. Siemens, G., Long, P.: Penetrating the fog: Analytics in learning and education. vol. 46, pp. 30-32. EDUCAUSE, Boulder, CO, USA (2011)

16. Silius, K., Tervakari, A.M., Kailanto, M.: Visualizations of user data in a social media enhanced web-based environment in higher education. In: Global Engineering Education Conference. pp. 893-899. IEEE (2013)

17. Verbert, K., Duval, E., Klerkx, J., Govaerts, S., Santos, J.L.: Learning analytics dashboard applications. American Behavioral Scientist 57(10), 1500-1509 (2013)

18. Wolff, A., Zdrahal, Z., Nikolov, A., Pantucek, M.: Improving retention: Predicting at-risk students by analysing clicking behaviour in a virtual learning environment. In: Proceedings of the Third International Conference on Learning Analytics and Knowledge. pp. 145-149. LAK '13, ACM, New York, NY, USA (2013) 